\section{Microbiological cure times in acanthamoeba keratitis}

\begin{abstract}
Aims The purpose of this study was to estimate the duration of treatment necessary for sequential acanthamoeba laboratory tests from corneal scrapings to become negative, and to assess predictors that affect this duration period.

Methods We included all patients with at least one positive acanthamoeba culture or Giemsa stain at the F.I. Proctor Foundation Microbiology Laboratory from 1996 to 2009.
\end{abstract}

A parametric survival analysis was performed among patients with repeat cultures to assess significant predictors for extended clearance time. Simulations were performed to estimate clearance time in the entire patient population, assuming imperfect sensitivity. Results Thirty-seven patients with laboratory evidence of acanthamoeba had testing at 69 time points. The median clearance time among eyes with repeat cultures was 42.5 days (interquartile range (IQR) 22.0-82.0 days; unadjusted parametric model). Initial visual acuity was the only predictor significantly associated with clearance time in univariate analyses $(P<0.0001)$. Using initial visual acuity as a predictor for clearance time among the entire patient population, the estimated clearance time decreased to 38.7 days $(95 \%$ confidence interval (CI) 27.9-53.5 days). When the imperfect sensitivity of the culture technique was also taken into account, the estimated clearance time was 44.1 days (95\% CI 31.9-61.0 days).

Conclusion The duration of infection with acanthamoeba keratitis undergoing treatment has not been well characterized. In this report we estimate a median clearance time of approximately 6 weeks, with an IQR of 22-82 days.

Eye (2011) 25, 1155-1160; doi:10.1038/eye.2011.126; published online 3 June 2011
CE Oldenburg ${ }^{1}$, JD Keenan ${ }^{1,2}, \mathrm{~V}$ Cevallos $^{1}$, MF Chan ${ }^{1,2}$, NR Acharya ${ }^{1,2}$, BD Gaynor ${ }^{1,2}$, SD McLeod ${ }^{1,2}$, EJ Esterberg ${ }^{1}$, TC Porco ${ }^{1,3}$ and TM Lietman ${ }^{1,2,3}$

Keywords: acanthamoeba; keratitis; survival analysis; cornea

\section{Introduction}

Acanthamoeba keratitis is difficult to treat, with minimal compelling evidence to guide decisions. ${ }^{1,2}$ Factors associated with poor outcome include delay in diagnosis, corticosteroid use before diagnosis, stromal involvement, ring infiltrate, and poor visual acuity at presentation. ${ }^{3-7}$ Treatment duration and follow-up are generally long, lasting till months. The persistence of corneal infection after initiation of appropriate therapy has not been well characterized. The purpose of this study is to estimate the time to microbiological cure in a treated patient population in which sequential cultures were available, and to assess predictors that may affect this time, including initial visual acuity, age, duration of symptoms before presentation, gender, corticosteroid use before presentation, and stromal involvement.

\section{Materials and methods}

The F.I. Proctor Foundation Microbiology Laboratory has extensive experience in detecting evidence of acanthamoeba using culture and smear. Most samples come from the clinics of the F.I. Proctor Foundation and University of California, San Francisco, but the laboratory also receives specimens from community ophthalmologists. For this study, we included all patients from the F.I. Proctor Foundation microbiology laboratory database who had at least one corneal Giemsa stain or were culture positive for acanthamoeba between July 1996 and September 2009. Patients with a clinical diagnosis but negative smears and cultures were not included. Neither confocal microscopy nor culture/smear of
${ }^{1}$ Francis I. Proctor Foundation, University of California San Francisco, San Francisco, CA, USA

${ }^{2}$ Department of Ophthalmology, University of California San Francisco, San Francisco, CA, USA

${ }^{3}$ Department of Epidemiology and Biostatistics, University of California San Francisco, San Francisco, CA, USA

Correspondence: JD Keenan, Francis I. Proctor Foundation, Room S338C, 513 Parnassus Avenue, University of CaliforniaSan Francisco,

San Francisco, CA 94143-0412, USA Tel: + 1415476 6323; Fax: +1 4154760527 E-mail: jeremy.keenan@ ucsf.edu

Received: 15 September 2010

Accepted in revised form: 11 April 2011 Published online: 3 June 2011 
contact lens cases had a role in diagnosis for this study. Non-nutrient agar plates with Escherichia coli overlay were used as the culture medium for acanthamoeba; growth was detected from the observation of motile trophozoites under microscopy. Giemsa stains were defined as positive when characteristic cysts consistent with acanthamoeba were observed. ${ }^{8-10}$ All cultures and stains were performed by an experienced microbiologist. Microbiological studies (stains and cultures) were obtained for all corneal ulcers in which infection with acanthamoeba was suspected. Following a positive acanthamoeba culture/stain and initiation of treatment, follow-up microbiological studies were obtained at the discretion of the physician, generally in the case of clinical suspicion of persistent infection or to confirm there was no longer active infection. Thus, the schedule of repeat cultures was not standardized. The medical records of each patient were reviewed to assess history and clinical signs from presentation to 6 months. Institutional review board approval was obtained from the UCSF Committee on Human Research.

Characteristics of individuals who had repeat cultures for acanthamoeba were compared with those of individuals without repeat cultures. Dichotomous variables (gender, occurrence of penetrating keratoplasty) were compared using Fisher's exact test. Continuous variables ( $\log$ MAR visual acuity at baseline and at approximately 6 months from presentation, duration of symptoms before diagnosis) were compared using the Wilcoxon rank-sum test.

Weibull regression with interval censoring ${ }^{11,12}$ was used to estimate the time to microbiological cure for ocular acanthamoebal infection, using the following univariate predictors: age, gender, steroids on presentation, duration of symptoms, visual acuity, and stromal involvement (as a binary indicator). Cases whose last culture remained positive were treated as rightcensored. This analysis was necessarily restricted to those patients in whom multiple cultures or smears were performed. The univariate regression using visual acuity as a covariate yielded an estimate of the entire probability distribution of clearance times for patients with any given visual acuity (and so on for each of the other univariate models). We determined which univariate predictors were statistically significant following Holm correction for multiple comparisons, and used these statistically significant univariate predictors in the subsequent analysis.

We applied the estimated Weibull clearance time distribution for any significant univariate predictors to the entire population (both patients who had multiple cultures, and those who did not). To do this, we estimated the distribution of the univariate predictor for the entire patient population and integrated the
Weibull clearance time distribution conditional on this estimated distribution. Finally, given this overall modeled distribution of clearance times, the median (and quantiles) were estimated by determining the value of the clearance time for which the cumulative distribution function (given the estimated parameters) equaled 0.5 (or the appropriate quantile). Approximate standard errors were computed using the delta method. ${ }^{13}$

We computed the survival curve by assuming a uniform distribution of true recovery times within the period between the last known positive test and the following negative test. For display, we integrated over the recovery time distribution (assumed to be uniform within the interval). ${ }^{14}$ At each point in time, we estimated the $95 \%$ confidence interval (CI) by non-parametric bootstrap. ${ }^{15}$ In addition, we conducted Weibull regression based on an assumption that the test sensitivity was $<1$, using adjusting for any significant univariate predictors from the original model (see Appendix for a detailed explanation of methods). All analyses were conducted with $\mathrm{R}$ version 2.12 (The R Foundation for Statistical Computing, www.r-project.org, Vienna, Austria).

\section{Results}

There were 37 patients with laboratory-confirmed acanthamoeba keratitis diagnosed at the F.I. Proctor Foundation microbiology laboratory from July 1996 to September 2009, with laboratory testing at 69 time points. ${ }^{16}$ We had access to the medical records for $32(86 \%)$ of these patients. Laboratory testing was repeated in 15 patients, with 8 patients receiving 1 repeat culture, 4 patients receiving 2 repeat cultures, and 1 patient each receiving 4, 5 , and 7 repeat cultures. Patients received repeated culture because they were not improving $(N=7,47 \%)$ or because the treating physician wanted to reduce medications because of toxicity or resolving disease $(N=8,53 \%)$. Of the 15 patients with repeated cultures, 10 had a subsequent culture negative for acanthamoeba. Of these patients, six $(60 \%)$ showed signs of healing within 1 month after negative culture, including reepithelialization, reduced inflammation, and symptomatic improvement. Three patients were not improving by 1 month after the negative culture, and 1 patient was lost to follow-up. Of five patients that required penetrating keratoplasty, three were performed at our institution - none of which showed acanthamoeba on pathology.

\section{Clinical characteristics}

Table 1 compares the clinical characteristics from the 17 eyes of 17 patients with a single positive culture with the 15 eyes of 15 patients who had more than one culture. 
Table 1 Characteristics of patients and eyes with culture or smear positive acanthamoeba keratitis

\begin{tabular}{|c|c|c|c|}
\hline & Repeated cultures/smear $(\mathrm{N}=15)$ & Single culture/smear $(\mathrm{N}=17)$ & P-value ${ }^{\mathrm{a}}$ \\
\hline Age, years (mean (range)) & $43.7(15-87)$ & $33.5(13-59)$ & 0.08 \\
\hline Female $(N,(\%))$ & $9(60 \%)$ & $12(71 \%)$ & 0.71 \\
\hline Presenting visual acuity (median (IQR)) & $20 / 70(20 / 50$ to $\mathrm{CF})$ & $20 / 70(20 / 30$ to $20 / 200)$ & 0.37 \\
\hline 6-month visual acuity ${ }^{\mathrm{b}}$ (median (IQR)) & CF $(20 / 125$ to $\mathrm{HM})$ & $20 / 25(20 / 20$ to $20 / 40)$ & 0.0003 \\
\hline $\begin{array}{l}\text { Duration of symptoms prior to presentation, days } \\
\text { (median (IQR)) }\end{array}$ & $30(14,56)$ & $23(17,30)$ & 0.66 \\
\hline Use of steroids at presentation (\%) & $12(80)$ & $14(82)$ & 1.0 \\
\hline Stromal involvement (\%) & $13(86.7)$ & $13(76.5)$ & 0.66 \\
\hline Penetrating keratoplasty (\%) & $5(35.7)$ & 0 & 0.01 \\
\hline Monotherapy (\%) & $4(26.7)$ & $8(47.1)$ & 0.29 \\
\hline
\end{tabular}

Abbreviations: $N$, number of patients (each patient had unilateral disease); $\mathrm{CF}$, counting fingers; $\mathrm{HM}$, hand motions; IQR, interquartile range.

aRank-sum test for continuous variables, and Fisher's exact test for categorical variables.

befined as visual acuity closest to the 6-month visit after presentation.

Of the 15 eyes with more than one culture, 11 eyes $(73 \%)$ received combination therapy with propamidine and either polyhexamethylene biguanide or chlorhexidine, 5 of which also received itraconazole or fluconazole. Four eyes $(27 \%)$ were treated with polyhexamethylene biguanide or chlorhexidine as monotherapy. Two of the 15 patients (13\%) were on anti-acanthamoebal drugs at presentation; one was on polyhexamethylene biguanide as monotherapy and one received combination therapy with polyhexamethylene biguanide and propamidine. We found no evidence that patients with repeated cultures had differing initial visual acuity than patients without repeated cultures $(P=0.37$, Wilcoxon rank-sum test), although the final visual acuity was worse in patients with repeated cultures $(P<0.001$, Wilcoxon rank-sum test). We found no evidence that duration of symptoms before diagnosis differed between the two groups ( $P=0.66$, Wilcoxon rank-sum test). Penetrating keratoplasty was more frequent among patients with repeat cultures $(P=0.01$, Fisher test; Table 1$)$.

\section{Predictors}

In univariate Weibull regression, initial best-corrected visual acuity (BCVA) was the only predictor tested that was significantly associated with microbial clearance time (relative clearance time $=1.10$ per Snellen line $(0.1$ $\log$ MAR), $P<0.0001$; Table 2). No evidence was found that gender $(P=0.29)$, age $(P=0.92)$, the use of corticosteroid eye drops at presentation $(P=0.23)$, or stromal involvement $(P=0.58)$ was associated with clearance time.

\section{Clearance time estimates}

When clearance time was modeled for the 15 eyes that had repeat cultures using a survival analysis adjusted for initial visual acuity, the estimated median time to
Table 2 Predictors of clearance time as detected by culture and/or smear

\begin{tabular}{llc}
\hline Explanatory variable & $\begin{array}{c}\text { Relative clearance }^{\text {time }} \text { (95\% CI) } \\
\text { P-value }\end{array}$ & \\
\hline Older age, per decade & $1.02(0.17,6.2)$ & 0.94 \\
Male gender & $1.95(0.57,6.7)$ & 0.29 \\
Corticosteroid use at presentation & $2.46(0.56,10.8)$ & 0.23 \\
Longer duration of symptoms, per & $0.79(0.10,5.96)$ & 0.82 \\
100 days & $1.10(1.05,1.16)$ & $<0.0001$ \\
$\begin{array}{l}\text { Worse initial visual acuity, per } \\
\text { Snellen line (0.1 logMAR) }\end{array}$ & $1.84(0.22,15.4)$ & 0.58 \\
\hline
\end{tabular}

Abbreviation: $\mathrm{CI}$, confidence interval.

${ }^{a}$ According to univariate parametric survival analyses predicting acanthamoebal clearance time.

microbial clearance was 42.5 days (95\% CI 22.0-82.0 days), with estimated interquartile range (IQR) of 25.5 days (95\% CI 19.5-33.3 days) to 88.5 days (95\% CI 21.4-365.7 days; Figure 1). When this model was performed for the entire patient population ( $n=32$ eyes), the estimated median clearance time decreased to 38.7 days (95\% CI 27.9-53.5 days). When we accounted for the imperfect sensitivity of culture and smear techniques for acanthamoeba diagnosis (assuming $83 \%$ sensitivity based on a previous study) ${ }^{17}$ the estimated clearance time increased to 44.1 days (95\% CI 31.9-61.0 days). In this model, when predicting duration of infection by baseline BCVA, patients who had a baseline acuity of Snellen 20/20 had a median duration of infection of 24.3 days (95\% CI 11.2-52.7 days), patients with a baseline acuity of Snellen 20/200 had a median duration of infection of 62.0 days (95\% CI 38.5-99.8 days), and patients with baseline acuity of count fingers had an estimated median duration of infection of 119.3 days (95\% CI 71.1-200.5 days). Finally, if we assumed that 


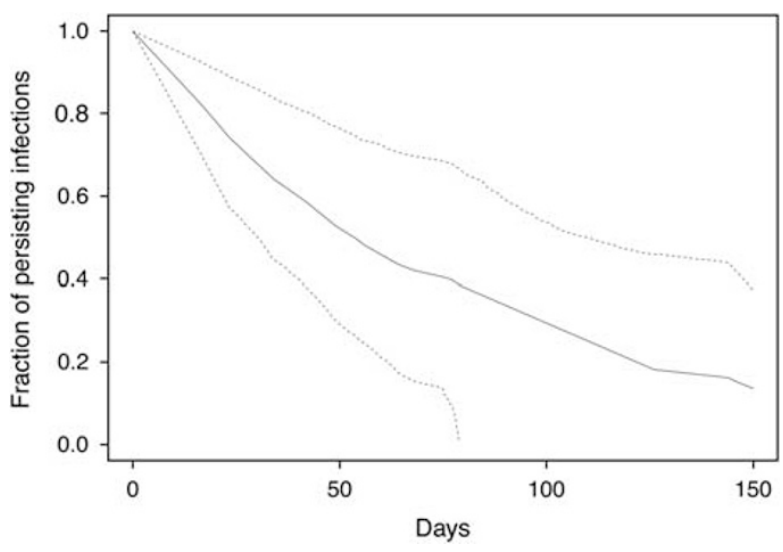

Figure 1 Survival curve for acanthamoeba clearance. Estimated survival curve (black line) with 95\% confidence interval (dashed lines) for the proportion of cases, which would still test positive at each time point.

the sensitivity of acanthamoeba culture and smear techniques was even lower (eg, sensitivity $=60 \%$ ), the estimated clearance time increased to 52.7 days (95\% CI 38.2-72.7 days). Still lower sensitivities would result in even longer estimations of the duration of infection.

\section{Discussion}

In a series of patients with laboratory-proven acanthamoeba infection and a subsequent corneal swabbing performed, the median microbial clearance time was 6-7 weeks in our unadjusted model, with an IQR of approximately 4-12 weeks. Better initial visual acuity was significantly associated with shorter microbial clearance times. The use of corticosteroids before presentation and stromal involvement appeared to lengthen the duration of infection, though these associations did not reach statistical significance.

The long duration of corneal infection in this study is consistent with other reports. A previous study noted viable acanthamoeba organisms as long as 23 months after initial presentation in a severe case, non-responsive to anti-acanthamoebal therapy. ${ }^{18}$ Treatment durations have been reported to last approximately 13 weeks; however, to our knowledge, median duration of active infection has never been characterized. ${ }^{19}$ In our patient population, the IQR of clearance times was 22-82 days (approximately 4 to 12 weeks), indicating that by 13 weeks of treatment, at least $75 \%$ of our patients would have cleared their infection.

We recognize that the median microbial clearance time from the unadjusted survival analysis is likely biased, as patients with worse disease would be more likely to have been recultured. Indeed, the patients recultured in our series had worse 6-month visual acuity than those patients not recultured and were more likely to require a penetrating keratoplasty. Therefore, we created a model to predict clearance time, given significant predictive factors as determined by univariate analyses, and used this model for all cases, whether they had been cultured more than once or not. This resulted in a decreased clearance time estimate of approximately 5-6 weeks for the single and repeat culture groups combined. However, these results should be interpreted with caution, as they were derived from a specific model of clearance time. An unbiased estimate of clearance time would require that all patients with acanthamoeba keratitis be cultured at regular intervals, regardless of disease severity. In the absence of these data, we have attempted instead to account for the inherent biases in the data using statistical methods.

The sensitivity of acanthamoeba culture and smear are unknown, but even the combination of the two tests is almost certainly imperfect. ${ }^{17}$ In addition, initiation of anti-acanthamoebal therapy could reduce the sensitivity of culture and smear, as topical therapy might clear superficial infection but not deeper infection. In other words, some repeat cultures were likely false negatives, leading to an underestimate of the median clearance time. We estimated the clearance time, assuming that a portion of our repeat cultures had been misclassified as negative. This model found a slight increase in clearance time. The true-negative predictive value and sensitivity of culture and smear in our facility is unknown, hence, if the actual sensitivity of the test were lower than our assumed value of $83 \%,{ }^{17}$ the expected clearance time would be longer. In a sensitivity analysis to address this possibility, we found that if the sensitivity of smear and culture was reduced to $60 \%$, the clearance time increased by approximately 10 days. If the actual sensitivity of smear and culture were $<60 \%$, the clearance time estimates would increase more; in fact, if the sensitivity of culture were in reality as low as $10 \%$, as has been claimed in reports using confocal as a gold standard, ${ }^{1}$ then our method would be unable to estimate a clearance time. However, it should be noted that culture-negative cases diagnosed with confocal clear, on average, quicker than culture-positive cases. ${ }^{1}$ Future studies could examine the expected clearing time of cysts on confocal.

A weakness of culture and smear as diagnostic techniques for acanthamoeba is that they may not identify stromal cysts too deep to be collected by swabbing. Confocal microscopy might be able to identify many more cases of acanthamoeba keratitis than are identified by culture and smear. ${ }^{1}$ The analysis did not include diagnoses made with confocal microscopy, a technique, which in theory allows the identification of deeper stromal cysts, and could increase the overall 
sensitivity to detect acanthamoebal infection, and potentially extend the estimated clearance time. ${ }^{7,17,20,21}$ This study should be repeated with confocal microscopy to determine if the clearance time estimates change with different diagnostic techniques. Identification of acanthamoeba infection might also depend on the quantity of organisms, which we did not take into account in our statistical analyses.

Worse initial BCVA was significantly associated with longer clearance times in a univariate model. In this model, each Snellen line (0.1 logMAR) corresponds to approximately a $10 \%$ increase in clearance time. Patients who present with worse BCVA likely have been infected for longer and have more severe disease than those who have better initial BCVA, and may therefore require more time to clear the infection. In a prediction model, patients with excellent vision at baseline $(20 / 20)$ had shorter clearance times than patients with worse vision at baseline.

The prior use of topical corticosteroids appeared to lengthen the time until acanthamoebal clearance, though this association was not statistically significant. Our observation is nevertheless consistent with other reports, which have shown that use of topical corticosteroids before acanthamoeba diagnosis and treatment may lengthen the duration of infection. ${ }^{7,22,23}$ We also found that eyes with stromal involvement at presentation seemed to have longer microbial clearance times compared with those without stromal involvement, though this too lacked statistical significance. This finding is consistent with previous studies that have shown an association between deep stromal keratitis and worse disease outcomes. ${ }^{3,7}$

In this report, we estimate that the median clearance time of acanthamoeba from corneas treated with standard anti-acanthamoebal medications is 42.5 days (IQR 22-82 days). This indicates that 50\% of our cases cleared between 4 and 12 weeks, though clearly the duration of treatment should be tailored for each individual case. Decreased visual acuity at presentation was significantly associated with a prolonged duration until microbial clearance. The duration of acanthamoebal infection was longer in those treated with topical corticosteroids compared with those who were not, though this association was not significant. This study provides evidence supporting the use of extended treatment protocols, especially for those presenting with decreased vision. The median clearance time found in this study is shorter than treatment durations typically reported in the literature. ${ }^{19}$ Note that this estimate is of the median, and that $50 \%$ of cases would be longer than 42 days. Because of this, it would be prudent to give antimicrobial therapy for longer than the median clearance time. As clearance is apparently correlated with severity, treatment duration may be tailored according to the response of each individual patient. Limitations of this study include a small sample size, and that there was likely a bias in our estimates. However, we attempted to correct for bias due to case selection or misclassification error. A larger, prospective study could be carried out to confirm the findings of this study, and might include confocal microscopy to allow identification of stromal infection with a sterile surface. In addition to clinical healing time and visual acuity, time to microbiological cure could be considered as an outcome for future clinical trials of antiparasitic agents.

\section{Summary}

\section{What was known before}

- Acanthamoeba keratitis is a rare but serious infection of the cornea that can potentially result in blindness.

- Duration of treatment and follow-up is generally very long, lasting till months.

- Factors associated with poor outcome include delay in diagnosis, corticosteroid use before diagnosis, stromal involvement, ring infiltrate, and poor visual acuity at presentation.

\section{What this study adds}

- Active organisms remain persistent in the cornea for a long period of time; in this study we found an interquartile range of 22-82 days for duration of active infection with appropriate treatment.

- Worse initial baseline visual acuity was significantly associated with increased duration of infection, indicating that these patients may have more severe disease requiring longer treatment duration to clear the infection.

\section{Conflict of interest}

The authors declare no conflict of interest.

\section{References}

1 Mathers W. Acanthamoeba: a difficult pathogen to evaluate and treat. Cornea 2004; 23(4): 325.

2 Roberts C, Henriquez F. Drug target identification, validation, characterisation and exploitation for treatment of Acanthamoeba (species) infections. Exp Parasitol 2010; 126(1): 91-96.

3 Claerhout I, Goegebuer A, Broecke VVD, Kestelyn P. Delay in diagnosis and outcome of Acanthamoeba keratitis. Graef's Arch Clin Exp Ophthalmol 2004; 242: 648-653.

4 Dart J, Saw V, Kilvington S. Acanthamoeba keratitis: diagnosis and treatment update 2009. Am J Ophthalmol 2009; 148: $487-499$

5 Illingworth C, Cook S, Karabatsas C, Easty D. Acanthamoeba keratitis: risk factors and outcome. Br J Ophthalmol 1995; 79: 1078-1082.

6 Ueki N, Eguchi H, Oogi Y, Shiota H, Yamane S, Umazume H et al. Three cases of Acanthamoeba keratitis diagnosed and treated in the early stage. J Med Invest 2009; 56: 166-169. 
7 Tu E, Joslin C, Sugar J, Shoff M, Booton G. Prognostic factors affecting visual outcome in Acanthamoeba keratitis. Ophthalmology 2008; 115: 1998-2003.

8 Marciano-Cabral F. Acanthamoeba spp as agents of disease in humans. Clin Microbiol Rev 2003; 16(2): 273-307.

9 Murray P, Baron E, Jorgensen J, Pfaller M, Yolken R. Manual of Clinical Microbiology, 8th edn. American Society for Microbiology: Washington, DC, 2003.

10 Wilhelmus K, Liesegang T, Osato M, Jones D. Cumitech 13 A Laboratory Diagnosis of Ocular Infections. American Society for Microbiology: Washington, DC, 1994.

11 Kalbfleisch J, Prentice R. The Statistical Analysis of Failure Time Data. Wiley-Interscience: Hoboken, NJ, 2002.

12 Gail M, Benichou J. Encylopedia of Epidemiologic Methods. Wiley: New York, NY, 2000.

13 Stuart A, Ord K. Kendall's Advanced Theory of Statistics, 6th edn. Hodder Arnold: London, 1994.

14 Sun K. The Statistical Analysis of Interval-Censored Failure Time Data. Springer Verlag: New York, NY, 2006.

15 Efron B. Logistic regression, survival analysis and the Kaplan-Meier curve. J Am Stat Assoc 1988; 83(402): 414-425.

16 Sansanayudh W, Cevallos V, Porco T, Margolis T, Lietman T, Acharya N. Fusarium and Acanthamoeba keratitis: can a single centre detect outbreaks? Br J Ophthalmol 2008; 92(5): 720-721.

\section{Appendix}

\section{Statistical methods}

To model longitudinal culture results, assuming imperfect sensitivity $s$ of our diagnostic test, we assumed independence given the true status (cleared or not), and complete specificity (cultures are never positive for uninfected individuals). For clarity, we let $F\left(t, v ; a, b_{0}, b_{1}\right)$ denote the Weibull cumulative distribution function ${ }^{11}$ for the clearance time for a patient with visual acuity $v$, given the Weibull shape parameter $a$ and a log-scale parameter of $b_{0}+b_{1} v$ (so that the distribution function for the clearance time $F\left(t, v ; a, b_{0}, b_{1}\right)=\frac{a}{b(v)}\left(\frac{t}{b(v)}\right)^{a-1} \mathrm{e}^{-(t / b(v))^{a}}$ is with $\left.b(v)=e^{b_{0}+b_{1} v}\right)$. Specifically, for each patient $i$ $(i=1, \ldots, 15)$ with repeat cultures taken at times $t_{i j}$ after the baseline visit, we modeled the probability of the culture results $X_{i j}\left(j=1, \ldots, n_{i}\right)$, taken at times $t_{k}$ for patient $i$ as

$$
\begin{aligned}
& P\left(X_{i 1}=x_{i 1}, \ldots X_{i n_{i}}=x_{i n_{i}}\right) \\
& \quad=\sum_{k=0}^{n_{i}}\left(\prod_{j=1}^{k} s^{x_{i j}}(1-s)^{1-x_{i j}} \prod_{j=k+1}^{n_{i}}\left(1-x_{i j}\right)\left(F\left(t_{i, k+1} ; a, b_{0}, b_{1}\right)-F\left(t_{i, k} ; a, b_{0}, b_{1}\right)\right)\right)
\end{aligned}
$$

17 Tu E, Joslin C, Sugar J, Booton G, Shoff M, Fuerst P. The relative value of confocal microscopy and superficial corneal scrapings in the diagnosis of Acanthamoeba keratitis. Cornea 2008; 27(7): 764-772.

18 Perez-Santonja J, Kilvington S, Hughes R, Tufail A, Matheson M, Dart J. Persistently culture positive Acanthamoeba keratitis: in vivo resistance and in vitro sensitivity. Ophthalmology 2003; 110: 1593-1600.

19 Lim N, Goh D, Bunce C, Xing W, Fraenkel G, Poole T et al. Comparison of polyhexamethylene biguanide and chlorhexidine as monotherapy agents in the treatment of Acanthamoeba keratitis. Am J Ophthalmol 2008; 145: 130-135.

20 Parmar D, Awwad S, Petroll W, Bowman R, McCulley J, Cavanagh H. Tandem scanning confocal corneal microscopy in the diagnosis of suspected Acanthamoeba keratitis. Ophthalmology 2006; 113: 538-547.

21 Winchester K, Mathers W, Sutphin J, Daley T. Diagnosis of Acanthamoeba keratitis in vivo with confocal microscopy. Cornea 1995; 14(1): 10-17.

22 McClellan K, Howard K, Niederkorn J, Alizadeh H. Effect of steroids on Acanthamoeba cysts and trophozoites. Invest Ophthalmol Vis Sci 2001; 42(12): 2885-2893.

23 John T, Lin J, Sahm D, Rockey J. Effects of corticosteroids in experimental acanthamoeba keratitis. Rev Infect Dis 1991; 13(Suppl 5): S440-S442.

where by convention $t_{i, 0}=0, t_{i, n_{i}+1}=\infty$, and each product is 1 when the index set is empty. Maximizing the likelihood provided estimates for $a, b_{0}$, and $b_{1}$; standard errors were derived from the observed information matrix. ${ }^{11}$ For patients with any given baseline acuity $v$, plugging in the estimated values for $a, b_{0}$, and $b_{1}$ yields an estimated clearance time distribution $F\left(t, v ; \hat{a}, \hat{b}_{0}, \hat{b}_{1}\right)$. We applied these estimates to the entire population by fitting a distribution $G(v)$ (specifically, a mixture of a beta distribution ${ }^{13}$ for visual acuities below (better than) 1.6 logMAR and a discrete distribution for acuities worse than 1.6) to the baseline visual acuity; integrating with respect to this distribution yielded an estimated population distribution of clearance times $H\left(t ; \hat{a}, \hat{b}_{0}, \hat{b}_{1}\right)=\int_{0}^{\infty} F\left(t, v ; \hat{a}, \hat{b}_{0}, \hat{b}_{1}\right) d G(v)$. 